\title{
The Arc Teeth Semi-rolled Cylindrical Gear Meshing Geometry
}

\author{
Vladimir Syzrantsev, Ksenia Syzrantseva
}

Industrial University of Tyumen, 38 Volodarskogo St., 625000 Tyumen, Russia

E-mail: syzrantsevvn@tyuiu.ru,syzrantsevakv@tyuiu.ru

\begin{abstract}
Arc teeth semi-rolled cylindrical gears in the conditions of unbraced machine bodies have a higher load capacity, durability and reliability as well as the ability to compensate for the shaft axis twist angle by self-adjustment of one of the wheels compared to straight and helical teeth gears. In the article, the study object is the arc teeth semirolled cylindrical gear. The wheel arc teeth are cut using the single division method without generating with the cutting head, the generating surface of which is a straight circular cone. The gear arc tooth flank is an envelope of the wheel teeth flanks family at a given relative movement. The semi-rolled version of arc teeth cylindrical gears allows to significantly simplify the technological process of cutting wheels and producing gears with large gear ratios. Mathematical models of wheel and pinion arc teeth flanks forming processes have been built for a semi-rolled cylindrical gear. Dependences for calculating principal curvatures of the wheel and pinion arc teeth flanks have been obtained. An algorithm and a program for calculating the coordinates of the points of the active path of action in arc teeth meshing and principal relative curvatures at these points have been developed. The problem of determining the size of the contact pattern in the studied gear has been solved. Calculations to estimate variations in the position of the active paths of action and the sizes of the contact pattern with varying the wheel and pinion axes twist angle and variations of the principal relative curvatures in the longitudinal and profile directions of the arc teeth at the points of active paths of action have been performed.
\end{abstract}

Keywords: semi-rolled cylindrical gear; arc teeth; teeth flank curvatures; lines of action; principal relative curvatures; contact pattern

\section{Introduction}

Cylindrical straight, helical and double helical teeth gears are the basis of most modern machines and mechanisms. The theoretical meshing contact in these gears occurs along the line. In the transmissions of tractors, locomotives, coal-mining and other energy-intensive machines, cylindrical gears are mounted on projecting shafts. In gear operations, deformations of shafts and bodies lead to misalignment of teeth in meshing, their edge contact, and a multiple reduction in the service life 
of gears. An effective way to increase the loading capacity and durability of cylindrical gears under the specified operating conditions is to use arc teeth (Figure 1). Arc teeth meshing may provide a linear, locally linear and point-topoint contact of the tooth flanks. Optimization of the geometry of these gears makes it possible to skip shifting the contact area to the edge of the tooth in the gear operation under teeth misalignment. The increased bending strength of arc teeth and the possibility of compensating for the twist (misalignment) angle of the teeth by self-adjustment of one of the transmission elements indicate the effectiveness of using arc teeth cylindrical gears in the drives of modern energysaturated machines.

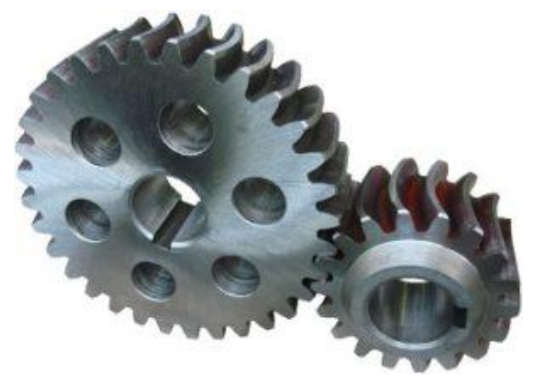

Figure 1

Arc teeth cylindrical gears

Currently, a number of methods are proposed for cutting generating cylindrical gear wheel arc teeth, which differ in the used tools and forming movements [1, 2, $3,4]$. The helix angle of the arc tooth in its midsection is equal to zero. Arc teeth cylindrical gear meshing in the proposed methods is based on the counterpart rack. In $[1,5,6]$, variants of cutting cylindrical wheel arc teeth with circular cutting heads by means of generating with single division on $\mathrm{CNC}$ machines are considered.

The methods worked out for cutting spiral teeth of generating bevel gear wheels are the basis of most of the above methods [7, 8]. For mass production of bevel gears, it has become possible to reduce the cost of their manufacture by switching to a semi-rolled gear option $[9,10]$. The wheel arc teeth of such a gear are cut with a cutting head without generating at a single division. Gleason specialists have developed the FORMATE and HELIXFORM methods in this area. Despite the more difficult task of finding the optimal geometry $[9,10]$, the technology of manufacturing such bevel gears is more advanced.

The analysis of the works related to the study of geometry [1, 2, 3, 4], contact and bending strength and durability $[1,11,12]$ of arc teeth gears shows that all of them are dedicated to generating cylindrical gears. The models of forming arc teeth flanks for semi-rolled cylindrical gears are considered in the works [13,14]. Prior to this work, the issues of calculating the geometric characteristics of the arc teeth contact (the coordinates of the contact points in different meshing phases, the 
principal relative curvatures at the contact points, and the size of the contact pattern) in meshing of the semi-rolled cylindrical gear have not been considered. The noted geometric characteristics of arc teeth meshing are necessary for developing a calculation method for contact loading and load-bearing capacity by the value of the transmitted torque of arc teeth cylindrical gears.

\section{Forming the Semi-rolled Cylindrical Gear Wheel Arc Tooth Usable Flank}

According to the methods of forming semi-rolled bevel gears [9, 10], in the manufacture of semi-rolled cylindrical gears, the arc teeth of the wheel are cut by the single division method without generating with a cutting head, the generating surface of which is a straight circular cone. In this case, the usable flank of the wheel arc tooth will also be the surface of the straight circular cone. Forming the usable flank of the pinion arc tooth is implemented on the basis of the generating wheel. The flank of the arc pinion tooth is the envelope of the flank of the wheel tooth at a given relative movement of the wheel and pinion in the transmission. Modern four-axes CNC machines allow implementing this method of cutting arc pinion teeth $[5,6]$. The transmission formed according to this scheme, in the absence of errors in the relative position of the pinion and wheel, is matched (theoretically accurate).

We describe the generating surface of the cutting head (straight circular cone) in coordinate system $S_{p}\left(x_{p}, y_{p}, z_{p}\right)$ rigidly connected to it (Figure 2), as follows $[13,14]$ :

$x_{p}=\cos \vartheta\left(u \cdot \sin \alpha_{0}-r_{g 2}\right) ; y_{p}=u \cdot \cos \alpha_{0} ; z_{p}=\sin \vartheta\left(u \cdot \sin \alpha_{0}-r_{g 2}\right)$,

where: $u, \vartheta$ are linear and angular parameters of the generating surface; $\alpha_{0}$ is a basic profile angle; $r_{g 2}$ is a calculated radius of the cutting head rotating around axis $y_{p}$ of coordinate system $S_{p}\left(x_{p}, y_{p}, z_{p}\right)$.

Taking into account the way of forming the usable surface of the wheel tooth, radius-vector $\bar{r}_{p}\left(x_{p}, y_{p}, z_{p}\right)$ is to be written into the coordinate system $S_{2}\left(x_{2}, y_{2} z_{2}\right)$, rigidly connected to the wheel:

$\tilde{r}_{2}=\tilde{A}_{2, p} \cdot \tilde{r}_{p}$,

where: $\tilde{A}_{2, p}$ is a fourth-order matrix describing the transition from coordinate system $S_{p}\left(x_{p}, y_{p}, z_{p}\right)$ to system $S_{2}\left(x_{2}, y_{2} z_{2}\right)$, the elements of which are 
determined in accordance with Figure $3 ; \tilde{r}_{2}, \tilde{r}_{p}$ are columns matrixes made up of vector radii coordinates $\bar{r}_{2}$ and $\bar{r}_{p}$ respectively.

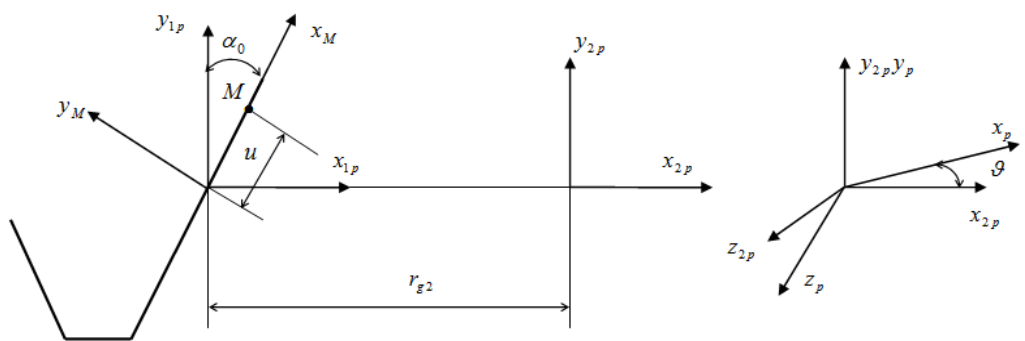

Figure 2

Cutting head generating surface

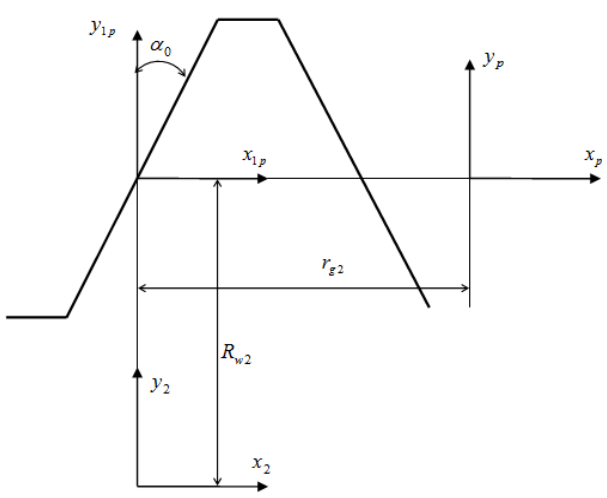

Figure 3

Coordinate systems to define elements of matrix $\widetilde{A}_{2, p}$

Matrix $\tilde{A}_{2, p}$ has the following form:

$$
\tilde{A}_{2, p}=\left\|\begin{array}{lllc}
1 & 0 & 0 & r_{g 2} \\
0 & 1 & 0 & R_{w 2} \\
0 & 0 & 1 & 0 \\
0 & 0 & 0 & 1
\end{array}\right\|
$$

where: $R_{w 2}$ is the radius of the pitch circle.

We find the projections of the wheel radius-vector $\bar{r}_{2}\left(x_{2}, y_{2}, z_{2}\right)$ based on (1) and (3), opening equation (2):

$x_{2}=\cos \vartheta\left(u \cdot \sin \alpha_{0}-r_{g 2}\right)+r_{g 2} ; y_{2}=u \cdot \cos \alpha_{0}+R_{w 2} ; z_{2}=\sin \vartheta\left(u \cdot \sin \alpha_{0}-r_{g 2}\right)$. 
Projections $m_{2 x}, m_{2 y}, m_{2 z}$ of the flank normal unitary vector (4) have the form:

$m_{2 x}=\cos \alpha_{0} \cdot \cos \vartheta ; \quad m_{2 y}=-\sin \alpha_{0} ; \quad m_{2 z}=\cos \alpha_{0} \cdot \sin \vartheta$.

Expressions (4) and (5) describe radius-vector $\bar{r}_{2}\left(x_{2}, y_{2}, z_{2}\right)$ and normal unitary vector $\bar{m}_{2}\left(m_{2 x}, m_{2 y}, m_{2 z}\right)$ of the usable flank of the convex side of the wheel arc tooth in coordinate system $S_{2}\left(x_{2}, y_{2} z_{2}\right)$.

From the theory of spatial gearing by meshing, it is known [7], that to determine the law of stress distribution over the contact area in meshing during the torque transmission, it is required to be able to calculate the gap at the point of contact of the teeth usable flanks. This gap is accurate to the values of the second order of smallness in main directions to determine the principal relative curvatures calculated at the point of contact of the tooth flanks. To calculate the principal curvatures of the arc teeth flanks and principal relative curvatures in meshing of the semi-rolled cylindrical gear, we use the methods developed in the theory of spatial gearing [7]. Following these methods, for the arc wheel teeth flanks, curvatures $(k)$ are determined based on the ratio:

$d \bar{m}_{2}=-k \cdot d \bar{r}_{2}$,

where: $\bar{m}_{2}$ is the normal unitary vector of the arc wheel tooth flank (5); $\bar{r}_{2}$ is a radius vector of the arc wheel tooth flank (4).

Differentials of vectors $\bar{m}_{2}$ and $\bar{r}_{2}$ by parameters $u$ and $\vartheta$ have the form:

$$
d \bar{m}_{2}=\bar{m}_{2 u} d u+\bar{m}_{2 \vartheta} d \vartheta ; d \bar{r}_{2}=\bar{r}_{2 u} d u+\bar{r}_{2 \vartheta} d \vartheta .
$$

Here, the indices $u$ and $\vartheta$ indicate the partial derivative by parameters $u$ and $\vartheta$, respectively.

Based on dependencies (7), we present expression (6) in the form:

$\bar{m}_{2 u} d u+\bar{m}_{2 \vartheta} d \vartheta=-k\left(\bar{r}_{2 u} d u+\bar{r}_{2 \vartheta} d \vartheta\right)$.

This vector equation is equivalent to two scalar equations since vectors $d \bar{r}_{2}$ and $d \bar{m}_{2}$ lie in the tangent plane. Projecting these vectors onto axes $x_{2}$ and $z_{2}$ of coordinate systems $S_{2}\left(x_{2}, y_{2} z_{2}\right)$ rigidly connected to the wheel, we get two scalar equations:

$$
m_{2 x u} d u+m_{2 x \vartheta} d \vartheta=-k\left(x_{2 u} d u+x_{2 \vartheta} d \vartheta\right) ; m_{2 z u} d u+m_{2 z \vartheta} d \vartheta=-k\left(z_{2 u} d u+z_{2 \vartheta} d \vartheta\right) .
$$

The system of two linear equations (9) is transformed to a quadratic equation, referred to $k$, of the following form:

$k^{2} \cdot A_{2}+k \cdot B_{2}+C_{2}=0$, 
where: $A_{2}=z_{2 u} x_{2 \vartheta}-z_{2 \vartheta} x_{2 u} ; B_{2}=z_{2 u} m_{2 x \vartheta}+x_{2 \vartheta} m_{2 z u}-m_{2 x u} z_{2 \vartheta}-m_{2 z \vartheta} x_{2 u}$;

$C_{2}=m_{2 z u} m_{2 x \vartheta}-m_{2 z \vartheta} m_{2 x u}$, indices $u$ and $\vartheta$ of projections on the axis of coordinate system $S_{2}\left(x_{2}, y_{2} z_{2}\right)$ of vectors $\bar{r}_{2}$ and $\bar{m}_{2}$ mean partial derivatives by parameters $u$ and $\vartheta$.

Differentiating expressions (4) and (5) by parameters $u$ and $\vartheta$, we define:

$$
\begin{aligned}
& x_{2 u}=\cos \vartheta \sin \alpha_{0} ; \quad z_{2 u}=\sin \vartheta \sin \alpha_{0} ; \quad x_{2 \vartheta}=-\sin \vartheta\left(u \cdot \sin \alpha_{0}-r_{g 2}\right) ; \\
& z_{2 \vartheta}=\cos \vartheta\left(u \cdot \sin \alpha_{0}-r_{g 2}\right) ; \quad m_{2 x u}=0 ; \quad m_{2 x \vartheta}=-\cos \alpha_{0} \sin \vartheta ; \quad m_{2 z u}=0 ; \\
& m_{2 z \vartheta}=\cos \alpha_{0} \cos \vartheta .
\end{aligned}
$$

Substituting these expressions in equation (10), after solving it, we obtain the dependences for the principal curvatures of the arc wheel flank:

$k_{21}=-\cos \alpha_{0} /\left(u \cdot \sin \alpha_{0}-r_{g 2}\right)$ and $k_{22}=0$.

At the calculated point of the wheel tooth flank $(u=0)$, formulas (11) are simplified:

$$
k_{21}=\cos \alpha_{0} / r_{g 2}, k_{22}=0 \text {. }
$$

\section{Forming the Semi-rolled Cylindrical Gearing Gear Arc Tooth Usable Flank}

To determine the usable flank of the concave side of the pinion arc tooth, we use the fact that it is a one-parameter envelope of the family of wheel tooth usable flanks in a given relative motion - the rotation of the pinion and wheel with constant gear ratio $i^{*}=z_{2}^{*} / z_{1}^{*}=$ const $; z_{1}^{*}, z_{2}^{*}$ are the numbers of the pinion and wheel teeth.

Using the methods of the spatial meshing theory [7, 13, 14, 15], we write the equation of the pinion tooth usable flank in the form:

$\tilde{r}_{1}\left(u, \vartheta, \varphi_{2}\right)=\tilde{A}_{1,2}\left(\varphi_{2}\right) \tilde{r}_{2}(u, \vartheta) ; f\left(u, \vartheta, \varphi_{2}\right)=0$.

Here: $\tilde{A}_{1,2}\left(\varphi_{2}\right)$ is a fourth-order matrix describing the transition from coordinate system $S_{2}\left(x_{2}, y_{2} z_{2}\right)$ to coordinate system $S_{1}\left(x_{1}, y_{1} z_{1}\right)$ rigidly connected to the pinion (Figure 4); $\varphi_{2}$ is the angle of the wheel rotation when forming the pinion tooth flank, associated with the angle of its rotation $\varphi_{1}$ through gear ratio $i^{*}$ :

$\varphi_{1}=i^{*} \cdot \varphi_{2}=\left(z_{2}^{*} \cdot \varphi_{2}\right) / z_{1}^{*}$. 
The meshing equation $[7,13]$ is the last written in (13).

Using Figure 4, we define elements $a_{i j}, i=\overline{1,4} ; j=\overline{1,4}$ of matrix $\tilde{A}_{1,2}\left(\varphi_{2}\right)$ :

$a_{11}=\cos \left(\varphi_{1}+\varphi_{2}\right) ; a_{12}=\sin \left(\varphi_{1}+\varphi_{2}\right) ; a_{13}=0 ; a_{14}=-a_{w s} \sin \varphi_{1} ;$

$a_{21}=-\sin \left(\varphi_{1}+\varphi_{2}\right) ; a_{22}=\cos \left(\varphi_{1}+\varphi_{2}\right) ; a_{23}=0 ; a_{24}=-a_{w s} \cos \varphi_{1} ;$

$a_{31}=a_{32}=a_{34}=a_{41}=a_{42}=a_{43}=0 ; a_{33}=a_{44}=1$,

where: $a_{w s}$ - is the center distance in the machine meshing of the pinion and the generating wheel.
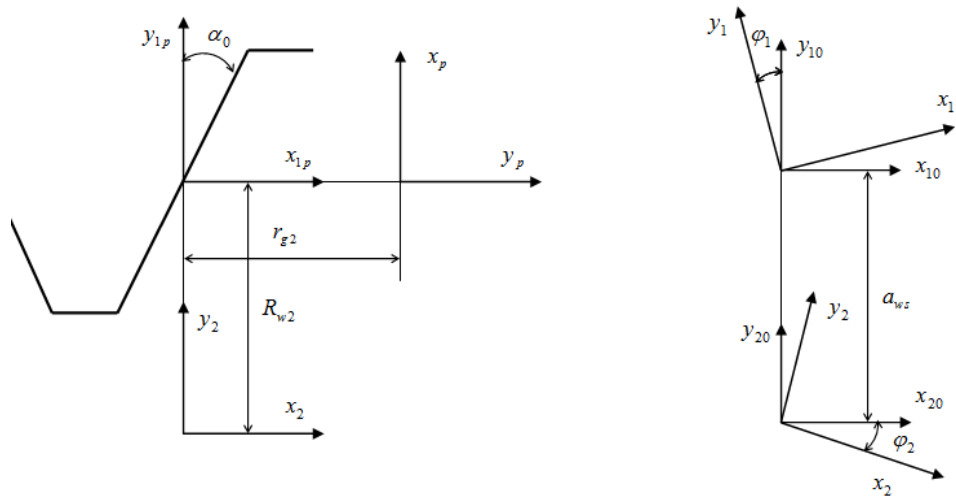

Figure 4

Coordinate systems to define elements of matrix $\tilde{A}_{1,2}\left(\varphi_{2}\right)$

Opening matrix equation (13) with respect to (4) and (15), we find the expressions for radius-vector $\bar{r}_{1}\left(x_{1}, y_{1}, z_{1}\right)$ projection of the usable flank of the pinion arc tooth:

$$
x_{1}=A \cdot \cos \left(\varphi_{1}+\varphi_{2}\right)+B \cdot \sin \left(\varphi_{1}+\varphi_{2}\right)-a_{w s} \cdot \sin \varphi_{1} ;
$$

$y_{1}=-A \cdot \sin \left(\varphi_{1}+\varphi_{2}\right)+B \cdot \cos \left(\varphi_{1}+\varphi_{2}\right)-a_{w s} \cdot \cos \varphi_{1} ;$

$z_{1}=\sin \vartheta\left(u \cdot \sin \alpha_{0}-r_{g 1}\right)$,

where: $A=\cos \vartheta\left(u \cdot \sin \alpha_{0}-r_{g 1}\right)+r_{g 1} ; B=u \cdot \cos \alpha_{0}+R_{2 w}$.

To obtain the meshing equation we use the method described in [13, 14], according to which, the meshing equation is written in the form:

$f\left(u, \vartheta, \varphi_{2}\right)=\bar{V}_{\varphi} \cdot \bar{m}_{2}=V_{\varphi x} \cdot m_{2 x}+V_{\varphi y} \cdot m_{2 y}+V_{\varphi z} \cdot m_{2 z}=0$,

where $\bar{V}_{\varphi}$ is the vector analog of the relative speed, $\bar{m}_{2}$ is the normal unitary vector of the generating wheel arc tooth flank (5). 
Projections $V_{\varphi x}, V_{\varphi y}, V_{\varphi z}$ of radius-vector $\bar{V}_{\varphi}$ are calculated according to the expression $[13,14]$ :

$\tilde{V}_{\varphi}=\tilde{C}_{\varphi} \cdot \tilde{r}_{2}$.

Here: $\tilde{C}_{\varphi}=\tilde{A}_{1,2}^{-1} \cdot \frac{d \tilde{A}_{1,2}}{d \varphi_{2}}$ is the fourth-order matrix of the relative speed analog; $\tilde{V}_{\varphi}$ is a column matrix composed of projections $V_{\varphi x}, V_{\varphi y}, V_{\varphi z}$.

After differentiating elements (15) of matrix $\tilde{A}_{1,2}\left(\varphi_{2}\right)$ by $\varphi_{2}$ with respect to (14) based on the expression for matrix $\tilde{C}_{\varphi}(18)$, we obtain the following formulas for its elements $c_{i j}, j=\overline{1,4}$ :

$c_{11}=0 ; \quad c_{12}=\left(1+i^{*}\right) ; \quad c_{13}=0 ; \quad c_{14}=-a_{w s} \cdot i^{*} \cdot \cos \varphi_{2} ;$

$c_{21}=-\left(1+i^{*}\right) ; \quad c_{22}=0 ; \quad c_{23}=0 ; \quad c_{24}=-a_{w s} \cdot i^{*} \cdot \sin \varphi_{2} ;$

$c_{31}=c_{32}=c_{33}=c_{44}=0 ; \quad c_{41}=c_{42}=c_{43}=c_{44}=0$.

Having the dependencies (19) and opening the matrix product (18) taking into account (4), we define expressions for radius-vector $\bar{V}_{\varphi}$ projections:

$V_{\varphi x}=\left(1+i^{*}\right) \cdot B-i^{*} \cdot a_{w s} \cdot \cos \varphi_{2} ; V_{\varphi y}=\left(1+i^{*}\right) \cdot B-i^{*} \cdot a_{w s} \cdot \sin \varphi_{2} ; V_{\varphi z}=0$,

substituting which in equation (17) jointly with expressions (5) and (16), we obtain the meshing equation in the following form:

$$
\begin{aligned}
& f\left(u, \vartheta, \varphi_{2}\right)=u \cdot \cos \vartheta \cdot\left(1+i^{*}\right)+\cos \alpha_{0} \cdot \cos \vartheta \cdot\left\lfloor R_{2 w} \cdot\left(1+i^{*}\right)-i^{*} \cdot a_{w s} \cdot \cos \varphi_{2}\right\rfloor+ \\
& +\sin \alpha_{0} \cdot\left\lfloor\left(1+i^{*}\right) \cdot r_{g 1} \cdot(1-\cos \vartheta)-i^{*} \cdot a_{w s} \cdot \sin \varphi_{2}\right\rfloor=0 .
\end{aligned}
$$

This equation can be presented as analytically resolved or with respect to parameter $u$ :

$$
u=-\frac{\cos \alpha_{0} \cos \vartheta\left[R_{2 w}\left(1+i^{*}\right)-i^{*} a_{w s} \cos \varphi_{2}\right\rfloor+\sin \alpha_{0}\left\lfloor\left(1+i^{*}\right) r_{g 1}(1-\cos \vartheta)-i^{*} a_{w s} \sin \varphi_{2}\right\rfloor}{\left(1+i^{*}\right) \cos \vartheta}
$$

or with respect to the parameter $\varphi_{2}$ :

$\varphi_{2}=\arcsin \left(-C_{u} / \sqrt{A_{u}^{2}+B_{u}^{2}}\right)-\xi$,

where: $A_{u}=-i^{*} \cdot a_{w s} \cdot \sin \alpha_{0} ; B_{u}=-i^{*} \cdot a_{w s} \cdot \cos \alpha_{0} \cdot \cos \vartheta ; \xi=\arcsin \left(B_{u} / \sqrt{A_{u}^{2}+B_{u}^{2}}\right)$;

$C_{u}=u \cdot \cos \vartheta \cdot\left(1+i^{*}\right)+\cos \alpha_{0} \cos \vartheta \cdot R_{w 2}\left(1+i^{*}\right)+\sin \alpha_{0}\left(1+i^{*}\right) \cdot r_{g 1} \cdot(1-\cos \vartheta)$. 
Dependences (16) jointly with equation (21) fully describe the geometry of the usable flank of the concave side of the arc pinion tooth (13).

Projections, $m_{1 x}, m_{1 y}, m_{1 z}$, of the normal unitary vector of the usable flank of the arc pinion tooth are determined based on the matrix equation:

$$
\tilde{m}_{1}\left(u, \vartheta, \varphi_{2}\right)=\tilde{A}_{1,2}\left(\varphi_{2}\right) \tilde{m}_{2}(u, \vartheta) .
$$

Opening the expression (24) based on dependences (15) and (5), we find:

$$
\begin{aligned}
& m_{1 x}=\cos \left(\varphi_{1}+\varphi_{2}\right) \cos \alpha_{0} \cos \vartheta-\sin \alpha_{0} \sin \left(\varphi_{1}+\varphi_{2}\right) ; \\
& m_{1 y}=-\sin \left(\varphi_{1}+\varphi_{2}\right) \cos \alpha_{0} \cos \vartheta-\sin \alpha_{0} \cos \left(\varphi_{1}+\varphi_{2}\right) ; \\
& m_{1 z}=\cos \alpha_{0} \sin \vartheta .
\end{aligned}
$$

We obtain formulas for calculating principal curvatures of the pinion arc tooth flank the way we did previously for the wheel arc tooth flank. To solve this problem, we use dependence (6) in the form:

$$
d \bar{m}_{1}=-k \cdot d \bar{r}_{1},
$$

where: $\bar{m}_{1}$ is the normal unitary vector of the arc pinion tooth flank $(25) ; \bar{r}_{1}$ is the radius-vector of the arc pinion tooth flank (16). The flank of the pinion tooth is the envelope of the family of wheel tooth flanks and depends on three parameters: $u$, $\vartheta$ and $\varphi_{2}$, associated by the meshing equation (21). In this case, the differentials of vectors $\bar{m}_{1}$ and $\bar{r}_{1}$ by parameters $u, \vartheta$ and $\varphi_{2}$ have the form:

$$
d \bar{m}_{1}=\bar{m}_{1 u} d u+\bar{m}_{1 \vartheta} d \vartheta+\bar{m}_{1 \varphi} d \varphi ; d \bar{r}_{1}=\bar{r}_{1 u} d u+\bar{r}_{1 \vartheta} d \vartheta+\bar{r}_{1 \varphi} d \varphi,
$$

and dependence (26) takes the form:

$$
\bar{m}_{1 u} d u+\bar{m}_{1 \vartheta} d \vartheta+\bar{m}_{1 \varphi} d \varphi=-k\left(\bar{r}_{1 u} d u+\bar{r}_{1 \vartheta} d \vartheta+\bar{r}_{1 \varphi}\right) .
$$

Vectors $d \bar{r}_{1}$ and $d \bar{m}_{1}$ lie in the tangent plane. We project them on axes $x_{1}$ and $z_{1}$ of coordinate system $S_{1}\left(x_{1}, y_{1} z_{1}\right)$ rigidly connected to the pinion, and take into account that parameters $u, \vartheta$ and $\varphi_{2}$ are associated by the meshing equation (21). As a result, we obtain the following system of three scalar equations:

$$
\begin{aligned}
& m_{1 x u} d u+m_{1 x \vartheta} d \vartheta+m_{1 x \varphi} d \varphi=-k\left(x_{1 u} d u+x_{1 \vartheta} d \vartheta+x_{1 \varphi} d \varphi\right) ; \\
& m_{z 1 u} d u+m_{1 z \vartheta} d \vartheta+m_{1 z \varphi} d \varphi=-k\left(z_{1 u} d u+z_{1 \vartheta} d \vartheta+z_{1 \varphi} d \varphi\right) ; \\
& f_{u} d u+f_{\vartheta} d \vartheta+f_{\varphi} d \varphi=0,
\end{aligned}
$$

where: $f_{u}, f_{\vartheta}, f_{\varphi}$ are partial derivatives of the meshing equation (21) by parameters $u, \vartheta$ and $\varphi_{2}$. 
Based on the third equation of the system (29), we have $d \varphi=-\left(f_{u} d u+f_{\vartheta} d \vartheta\right) / f_{\varphi}$, after which the system (29) is transformed to the form:

$$
\begin{aligned}
& f_{\varphi}\left(m_{1 x u}+k \cdot x_{1 u}\right) d u+f_{\varphi}\left(m_{1 x \vartheta}+k \cdot x_{1 \vartheta}\right)-\left(m_{1 x \varphi}+k \cdot x_{1 \varphi}\right)\left(f_{u} d u+f_{\vartheta} d \vartheta\right)=0 \\
& f_{\varphi}\left(m_{1 z u}+k \cdot z_{1 u}\right) d u+f_{\varphi}\left(m_{1 z \vartheta}+k \cdot z_{1 \vartheta}\right)-\left(m_{1 z \varphi}+k \cdot z_{1 \varphi}\right)\left(f_{u} d u+f_{\vartheta} d \vartheta\right)=0 .
\end{aligned}
$$

Following the theory of differential geometry, to determine the values of principal curvatures, it is necessary to equate the determinant of the system of equations (30) to zero

$$
\left.\mid \begin{array}{ll}
f_{\varphi}\left(m_{1 x u}+k \cdot x_{1 u}\right)-f_{u}\left(m_{1 x \varphi}+k \cdot x_{1 \varphi}\right) ; & f_{\varphi}\left(m_{1 x \vartheta}+k \cdot x_{1 \vartheta}\right)-f_{\vartheta}\left(m_{1 x \varphi}+k \cdot x_{1 \varphi}\right) \\
f_{\varphi}\left(m_{1 z u}+k \cdot z_{1 u}\right)-f_{u}\left(m_{1 z \varphi}+k \cdot z_{1 \varphi}\right) ; & f_{\varphi}\left(m_{1 z \vartheta}+k \cdot z_{1 \vartheta}\right)-f_{\vartheta}\left(m_{1 z \varphi}+k \cdot z_{1 \varphi}\right)
\end{array}\right)=0
$$

and solve the resulting quadratic equation relative to $k$.

After the transformations, equation (31) has the following form:

$$
w_{1} \cdot k^{2}+\left(w_{2}+w_{3}\right) \cdot k+w_{4}=0,
$$

where:

$$
\begin{aligned}
& w_{1}=\left|\begin{array}{lll}
f_{u} & f_{\vartheta} & f_{\varphi} \\
x_{1 u} & x_{1 \vartheta} & x_{1 \varphi} \\
z_{1 u} & z_{1 \vartheta} & z_{1 \varphi}
\end{array}\right| ; \quad w_{2}=\left|\begin{array}{ccc}
f_{u} & f_{\vartheta} & f_{\varphi} \\
m_{1 x u} & m_{1 x \vartheta} & m_{1 x \varphi} \\
z_{1 u} & z_{1 \vartheta} & z_{1 \varphi}
\end{array}\right| ; \\
& w_{3}=\left|\begin{array}{ccc}
f_{u} & f_{\vartheta} & f_{\varphi} \\
x_{1 u} & x_{1 \vartheta} & x_{1 \varphi} \\
m_{1 z u} & m_{1 z \vartheta} & m_{1 z \varphi}
\end{array}\right| ; \quad w_{4}=\left|\begin{array}{ccc}
f_{u} & f_{\vartheta} & f_{\varphi} \\
m_{1 x u} & m_{1 x \vartheta} & m_{1 x \varphi} \\
m_{1 z u} & m_{1 z \vartheta} & m_{1 z \varphi}
\end{array}\right| .
\end{aligned}
$$

Similar formulas can be obtained using the normal unitary vector and radiusvector of the generating surface, in this case, it is the radius-vector of the generating wheel surface (4) and its normal unitary vector (5). In this case, the calculations are simplified, and the coefficients of equation (32) have the form:

$$
\begin{aligned}
& w_{1}=\left|\begin{array}{ccc}
f_{u} & f_{\vartheta} & f_{\varphi} \\
x_{2 u} & x_{2 \vartheta} & V_{\varphi x} \\
z_{2 u} & z_{2 \vartheta} & V_{\varphi z}
\end{array}\right| ; \quad w_{2}=\left|\begin{array}{ccc}
f_{u} & f_{\vartheta} & f_{\varphi} \\
m_{2 x u} & m_{2 x \vartheta} & w_{\varphi x} \\
z_{2 u} & z_{2 \vartheta} & V_{\varphi z}
\end{array}\right| ; \\
& w_{3}=\left|\begin{array}{ccc}
f_{u} & f_{\vartheta} & f_{\varphi} \\
x_{2 u} & x_{2 \vartheta} & V_{\varphi x} \\
m_{2 z u} & m_{2 z \vartheta} & w_{\varphi z}
\end{array}\right| ; \quad w_{4}=\left|\begin{array}{ccc}
f_{u} & f_{\vartheta} & f_{\varphi} \\
m_{2 x u} & m_{2 x \vartheta} & w_{\varphi x} \\
m_{2 z u} & m_{2 z \vartheta} & w_{\varphi z}
\end{array}\right| .
\end{aligned}
$$


Here: $f_{u}, f_{\vartheta}, f_{\varphi}$ are partial derivatives of the meshing equation (21) by parameters $u, \vartheta$ and $\varphi_{2} ; x_{2 u}, x_{2 \vartheta}, z_{2 u}, z_{2 \vartheta}$ are partial derivatives by parameters $u$ and $\vartheta$ of the radius-vector projections (4) on axes $x_{2}$ and $z_{2}$ of coordinate system $S_{2}\left(x_{2}, y_{2}, z_{2}\right) ; V_{\varphi x}$ and $V_{\varphi z}$ are vector-analog projections (20) of the relative speed on axes $x_{2}$ and $z_{2} ; w_{\varphi x}, w_{\varphi z}$ are projections of the vectoranalog of the angular velocity on axes $x_{2}$ and $z_{2} ; m_{2 x u}, m_{2 x \vartheta}, m_{2 z u}, m_{2 z \vartheta}$ are partial derivatives by parameters $u$ and $\vartheta$ of projections of the normal unitary vector (5) of the surface (4).

Projections $w_{\varphi x}$ and $w_{\varphi z}$ are calculated based on the same expression (18):

$\tilde{w}_{\varphi}=\tilde{C}_{\varphi} \cdot \tilde{m}_{2}=\tilde{A}_{1,2}^{-1} \cdot \frac{d \tilde{A}_{1,2}}{d \varphi_{2}} \cdot \tilde{m}_{2}$

opening which on the basis of (19) and (5), we obtain:

$w_{\varphi x}=-\left(1+i^{*}\right) \sin \alpha_{0} ; w_{\varphi y}=-\left(1+i^{*}\right) \cos \alpha_{0} \cos \vartheta ; w_{\varphi z}=0$.

The dependences for calculating the remaining elements of the determinants (34) have the form:

$$
\begin{aligned}
& f_{u}=\left(1+i^{*}\right) \cos \vartheta ; f_{\varphi}=i^{*} \cdot a_{w s}\left(\cos \alpha_{0} \cos \vartheta \sin \varphi_{2}-\sin \alpha_{0} \cos \varphi_{2}\right) ; \\
& f_{\vartheta}=-u\left(1+i^{*}\right) \sin \vartheta-\cos \alpha_{0} \sin \vartheta\left[R_{w 2}\left(1+i^{*}\right)-i^{*} \cdot a_{w s} \cos \varphi_{2}\right\rfloor+\left(1+i^{*}\right) r_{g 1} \sin \alpha_{0} \sin \vartheta ; \\
& x_{2 u}=\cos \vartheta \sin \alpha_{0} ; \quad x_{2 \vartheta}=-\sin \vartheta\left(u \sin \alpha_{0}-r_{g 2}\right) ; \quad m_{2 x u}=0 ; \\
& m_{2 x \vartheta}=-\cos \alpha_{0} \sin \vartheta ; \quad z_{2 u}=\sin \vartheta \sin \alpha_{0} ; \quad z_{2 \vartheta}=\cos \vartheta\left(u \sin \alpha_{0}-r_{g 2}\right) ; \quad m_{2 z u}=0 ; \\
& m_{2 z \vartheta}=\cos \alpha_{0} \cos \vartheta ; \quad V_{\varphi x}=\left(1+i^{*}\right)\left(u \cos \alpha_{0}+R_{w 2}\right)-i^{*} \cdot a_{w s} \cdot \cos \varphi_{2} ; \quad V_{\varphi z}=0 .
\end{aligned}
$$

We open the expressions (34) with respect to (37) and find:

$$
\begin{aligned}
& w_{1}=f_{\vartheta} \cdot V_{\varphi x} \cdot z_{2 u}+f_{\varphi}\left(x_{2 u} \cdot z_{2 \vartheta}-x_{2 \vartheta} \cdot z_{2 u}\right)-f_{u} \cdot V_{\varphi x} \cdot z_{2 \vartheta} ; \\
& w_{2}=f_{\vartheta} \cdot w_{\varphi x} \cdot z_{2 u}-f_{\varphi} \cdot m_{2 x \vartheta} \cdot z_{2 u}-f_{u} \cdot w_{\varphi x} \cdot z_{2 \vartheta} ; \\
& w_{3}=m_{2 z \vartheta}\left(f_{\varphi} \cdot x_{2 u}-f_{u} \cdot V_{\varphi x}\right) ; \quad w_{4}=-f_{u} \cdot m_{2 z \vartheta} \cdot w_{\varphi x} .
\end{aligned}
$$

As a result, we solve the equation (32) based on (38) and obtain the following expression for calculation of principal curvatures of the pinion tooth flank:

the curvature by the length of the tooth is

$$
k_{11}=\left[-\left(w_{2}+w_{3}\right)-\sqrt{\left(w_{2}+w_{3}\right)^{2}-4 \cdot w_{1} \cdot w_{4}}\right] /\left(2 \cdot w_{1}\right) \text {. }
$$


the curvature by the profile of the tooth is

$$
k_{12}=\left[-\left(w_{2}+w_{3}\right)+\sqrt{\left(w_{2}+w_{3}\right)^{2}-4 \cdot w_{1} \cdot w_{4}}\right] /\left(2 \cdot w_{1}\right) .
$$

At the calculated point of the pinion tooth flank: $u=0, \vartheta=0$ and $\varphi_{2}=\varphi_{1}=0$. For these values the formulas (39) and (40) are simplified and have the form:

$$
k_{11}=-\cos \alpha_{0} / r_{g 2} ; \quad k_{12}=-\left(1+i^{*}\right)^{2} /\left(i^{*} \cdot a_{w s} \cdot \sin \alpha_{0}\right) .
$$

\section{Calculation of Contact Points Coordinates of Wheel and Pinion Arc Teeth Flanks in Meshing}

Meshing semi-rolled cylindrical gear arc teeth is matched in the absence of errors of manufacturing and the wheel and pinion relative position, based on the pinion teeth forming method. Teeth meshing occurs in linear contact conditions. To exclude the edge contact that occurs due to errors in manufacturing teeth, it is localized in the longitudinal direction of the tooth. The gear remains matched, and the contact of the active flanks of the pinion and wheel teeth occurs in the tooth transverse midsection where the helix angle is zero. In the case when the gear operates under errors in the relative position of the wheel and pinion, their meshing becomes approximate with the teeth point contact.

The problem of determining the coordinates of the contact points of the active flanks of wheel and pinion arc teeth, installed with errors in their relative position, is an inverse problem of the meshing theory $[7,9,14]$. Knowing the coordinates of the contact points is required to solve the problem of calculating the contact loading of the gear. To solve the inverse problem, expressions of the radii-vectors and normal unitary vectors of pinion and wheel arc teeth flanks are required.

Based on the dependences (4), (5), the projections of radius-vector $\bar{r}_{2}^{2}\left(u_{2}, \vartheta_{2}\right)$ and normal unitary vector $\bar{m}_{2}^{2}\left(\vartheta_{2}\right)$ of the arc wheel tooth flank in coordinate system $S_{2}\left(x_{2}, y_{2} z_{2}\right)$ (superscript "2" in the designation of vectors) rigidly connected to the wheel, have the form:

$$
\begin{aligned}
& x_{2}\left(u_{2}, \vartheta_{2}\right)=\cos \vartheta_{2}\left(u_{2} \cdot \sin \alpha_{0}-r_{g 2}\right)+r_{g 2} ; y_{2}\left(u_{2}\right)=u_{2} \cdot \cos \alpha_{0}+R_{w 2} ; \\
& z_{2}\left(u_{2}, \vartheta_{2}\right)=\sin \vartheta_{2}\left(u_{2} \cdot \sin \alpha_{0}-r_{g 2}\right), \\
& m_{2 x}\left(\vartheta_{2}\right)=\cos \alpha_{0} \cdot \cos \vartheta_{2} ; \quad m_{2 y}=-\sin \alpha_{0} ; \quad m_{2 z}\left(\vartheta_{2}\right)=\cos \alpha_{0} \cdot \sin \vartheta_{2} .
\end{aligned}
$$

where: $u_{2}, \vartheta_{2}$ are linear and angular parameters of the wheel tooth flank. 
Projections of radius vector $\bar{r}_{1}^{1}\left(u_{1}, \vartheta_{1}, \varphi_{1}\right)$ and normal unitary vector $\bar{m}_{1}^{1}\left(\vartheta_{1}, \varphi_{1}\right)$ of the pinion tooth flank in coordinate system $S_{1}\left(x_{1}, y_{1}, z_{1}\right)$ rigidly connected to the pinion, taking into account the formulas (16), (21) and (25), are described by the following expressions:

$$
\begin{aligned}
& x_{1}\left(u_{1}, \vartheta_{1}, \varphi_{1}\right)=A \cdot \cos \left(\varphi_{1}+\varphi_{2}\right)+B \cdot \sin \left(\varphi_{1}+\varphi_{2}\right)-a_{w s} \cdot \sin \varphi_{1} ; \\
& y_{1}\left(u_{1}, \vartheta_{1}, \varphi_{1}\right)=-A \cdot \sin \left(\varphi_{1}+\varphi_{2}\right)+B \cdot \cos \left(\varphi_{1}+\varphi_{2}\right)-a_{w s} \cdot \cos \varphi_{1} ; \\
& z_{1}\left(u_{1}, \vartheta_{1}\right)=\sin \vartheta_{1}\left(u_{1} \cdot \sin \alpha_{0}-r_{g 1}\right), \\
& f\left(u_{1}, \vartheta_{1}, \varphi_{1}\right)=u_{1} \cdot \cos \vartheta_{1} \cdot(1+i)+\cos \alpha_{0} \cdot \cos \vartheta_{1} \cdot\left[R_{2 w} \cdot(1+i)-i \cdot a_{w s} \cdot \cos \varphi_{2}\right]+ \\
& +\sin \alpha_{0} \cdot\left[(1+i) \cdot r_{g 1} \cdot\left(1-\cos \vartheta_{1}\right)-i \cdot a_{w s} \cdot \sin \varphi_{2}\right]=0, \\
& z_{2}\left(u_{2}, \vartheta_{2}\right)=\sin \vartheta_{2}\left(u_{2} \cdot \sin \alpha_{0}-r_{g 2}\right), \\
& m_{1 x}\left(\vartheta_{1}, \varphi_{1}\right)=\cos \left(\varphi_{1}+\varphi_{2}\right) \cos \alpha_{0} \cos \vartheta_{1}-\sin \alpha_{0} \sin \left(\varphi_{1}+\varphi_{2}\right) \\
& m_{1 y}\left(\vartheta_{1}, \varphi_{1}\right)=-\sin \left(\varphi_{1}+\varphi_{2}\right) \cos \alpha_{0} \cos \vartheta_{1}-\sin \alpha_{0} \cos \left(\varphi_{1}+\varphi_{2}\right) ; \\
& m_{1 z}\left(\vartheta_{1}\right)=\cos \alpha_{0} \cos \vartheta_{1} .
\end{aligned}
$$

where: $A=\cos \vartheta_{1}\left(u_{1} \cdot \sin \alpha_{0}-r_{g 1}\right)+r_{g 1} ; \quad B=u_{1} \cdot \cos \alpha_{0}+R_{2 w} ; \quad u_{1}, \quad \vartheta_{1}$ are linear and angular parameters of the pinion tooth flank; $\varphi_{1}=i^{*} \cdot \varphi_{2}=\left(z_{2}^{*} \cdot \varphi_{2}\right) / z_{1}^{*}$ is the angle of rotation of the pinion (14) during forming the arc tooth flank on the base of the generating wheel.

The movable links of the gear - the pinion and wheel - rotate around axes $z_{1}$, and $z_{2}$. The pinion and wheel are associated with coordinate systems $S_{1}\left(x_{1}, y_{1}, z_{1}\right)$ and $S_{2}\left(x_{2}, y_{2} z_{2}\right)$. We assume that the starting point of rotation angle $\psi_{k}$ of the $k^{\text {th }}$ movable link $(k=1,2)$ in operating meshing corresponds to the position of axis $y_{k},(k=1,2)$ in the axial plane of the gear. The relative position of the pinion and wheel in working meshing (in the absence of rotation) is set by center distance $a_{w p}$, which differs from the machine distance $\left(a_{w s}\right)$ by the value of $\pm \delta a_{w}$ and teeth twist angle $\gamma$.

To study semi-rolled cylindrical gear arc teeth meshing, we determine the position of coordinate system $S_{2}\left(x_{2}, y_{2} z_{2}\right)$ relative to system $S_{1}\left(x_{1}, y_{1}, z_{1}\right)$ using the fourth-order transition matrix $\tilde{D}_{1,2}\left(\psi_{1}, \psi_{2}\right)=\left\|d_{i, j}\right\|, i, j=\overline{1,4}$. The elements of this matrix have the form: 
$d_{11}=\cos \psi_{1} \cos \psi_{2}-\sin \psi_{1} \cos \gamma \sin \psi_{2} ; d_{12}=\cos \psi_{1} \sin \psi_{2}+\sin \psi_{1} \cos \gamma \cos \psi_{2} ;$

$d_{13}=\sin \psi_{1} \sin \gamma ; d_{14}=-a_{w p} \sin \psi_{1} ; d_{21}=-\sin \psi_{1} \cos \psi_{2}-\cos \psi_{1} \cos \gamma \sin \psi_{2} ;$

$d_{22}=-\sin \psi_{1} \sin \psi_{2}+\cos \psi_{1} \cos \gamma \cos \psi_{2} ; d_{23}=\cos \psi_{1} \sin \gamma$;

$d_{24}=-a_{w p} \cos \psi_{1} ; d_{31}=\sin \gamma \sin \psi_{2} ; d_{32}=-\sin \gamma \cos \psi_{2} ; d_{33}=\cos \gamma$;

$d_{34}=0 ; \quad d_{41}=d_{42}=d_{43}=0 ; \quad d_{44}=1$.

With $\gamma=\delta a_{w}=0$, the elements of matrix $\tilde{D}_{1,2}\left(\psi_{1}, \psi_{2}\right)$ (47) when $\psi_{1}$ is replaced by $\varphi_{1}$ and $\psi_{2}$ is replaced by $\varphi_{2}$ coincide with the elements of matrix $\tilde{A}_{1,2}\left(\varphi_{1}, \varphi_{2}\right)$ (15). If the function of changing positions of the gear parts

$\psi_{2}=\psi_{2}\left(\psi_{1}\right)$

at given values $\gamma$ and $a_{w p}$ is known, then matrix $\tilde{D}_{1,2}\left(\psi_{1}, \psi_{2}\right)$ describes the relative movement of the wheel and pinion during the gear operation. In the nonenveloping gear, the law of parameter $\psi_{2}$ variation is established after determining the contact points of the active flanks of the pinion and wheel teeth within the single-contact mesh. According to studies [7, 9, 14] of the meshing theory, the contact point on the active flank of the pinion tooth for a fixed value of its rotation angle $\left(\psi_{1}=\right.$ const $)$ is determined by solving the inverse meshing problem $[7,9,14]$, the mathematical description of which is the following system of equations:

$$
\begin{aligned}
& \tilde{r}_{1}^{1}\left(u_{1}, \vartheta_{1}, \varphi_{1}\right)=\tilde{D}_{1,2}\left(\psi_{1}, \psi_{2}\right) \tilde{r}_{2}^{2}\left(u_{2}, \vartheta_{2}\right) ; \\
& \tilde{m}_{1}^{1}\left(u_{1}, \vartheta_{1}, \varphi_{1}\right)=\tilde{D}_{1,2}\left(\psi_{1}, \psi_{2}\right) \tilde{m}_{2}^{2}\left(u_{2}, \vartheta_{2}\right) ; \quad f\left(u_{1}, \vartheta_{1}, \varphi_{1}\right)=0 .
\end{aligned}
$$

Here, the superscript defines the coordinate system in which the vector projections are calculated; $\tilde{r}_{2}^{2}, \quad \tilde{m}_{2}^{2}$ are columns matrixes made up of the coordinate projections of radius-vector $\bar{r}_{2}^{2}$ (42) and normal unitary vector $\bar{m}_{2}^{2}$ (43) of the wheel tooth active flank in coordinate system $S_{2} ; \tilde{r}_{1}^{1}, \tilde{m}_{1}^{1}$ are columns matrixes made up of the coordinate projections of radius-vector $\bar{r}_{1}^{1}$ (44) and normal unitary vector $\bar{m}_{1}^{1}$ (46) of the wheel tooth active flank in coordinate system $S_{1}$; $f\left(u_{1}, \vartheta_{1}, \varphi_{1}\right)=0$ is the equation of meshing in processing the pinion teeth flanks (45).

The system (49) corresponds to the conditions of the correct contact of the pinion and wheel teeth active flanks and is equivalent to six scalar transcendental equations (the equality of normal unitary vectors only gives two independent equations) with seven unknowns $u_{1}, \vartheta_{1}, \varphi_{1}, u_{2}, \vartheta_{2}, \psi_{1}, \psi_{2}$. 


$$
\begin{aligned}
& x_{1}\left(u_{1}, v_{1}, \varphi_{1}\right)=d_{11}\left(\psi_{1}, \psi_{2}\right) x_{2}\left(u_{2}, v_{2}\right)+d_{12}\left(\psi_{1}, \psi_{2}\right) y_{2}\left(u_{2}\right)+d_{13}\left(\psi_{1}\right) z_{2}\left(u_{2}, \vartheta_{2}\right)+d_{14}\left(\psi_{1}\right) ; \\
& y_{1}\left(u_{1}, \vartheta_{1}, \varphi_{1}\right)=d_{12}\left(\psi_{1}, \psi_{2}\right) x_{2}\left(u_{2}, v_{2}\right)+d_{22}\left(\psi_{1}, \psi_{2}\right) y_{2}\left(u_{2}\right)+d_{23}\left(\psi_{1}\right) z_{2}\left(u_{2}, \vartheta_{2}\right)+d_{24}\left(\psi_{1}\right) ; \\
& z_{1}\left(u_{1}, v_{1}\right)=d_{31}\left(\psi_{2}\right) x_{2}\left(u_{2}, v_{2}\right)+d_{32}\left(\psi_{2}\right) y_{2}\left(u_{2}\right)+d_{33}\left(\psi_{2}\right) z_{2}\left(u_{2}, \vartheta_{2}\right)+d_{34} ; \\
& m_{1 x}\left(v_{1}, \varphi_{1}\right)=d_{11}\left(\psi_{1}, \psi_{2}\right) m_{2 x}\left(v_{2}\right)+d_{12}\left(\psi_{1}, \psi_{2}\right) m_{2 y}+d_{13}\left(\psi_{1}\right) m_{2 z}\left(\vartheta_{2}\right) ; \\
& m_{1 z}\left(v_{1}\right)=d_{31}\left(\psi_{2}\right) m_{2 x}\left(v_{2}\right)+d_{32}\left(\psi_{2}\right) m_{2 y}+d_{33} m_{2 z}\left(\vartheta_{2}\right) ; f\left(u_{1}, \vartheta_{1}, \varphi_{1}\right)=0 .
\end{aligned}
$$

To determine the coordinates of the contact point of the pinion and wheel teeth flanks with specified errors of the relative position $\left(\delta a_{w}, \gamma\right)$ of the pinion and the wheel, it is sufficient to fix the meshing phase $\left(\psi_{1}=\right.$ const $)$ within the pinion tooth spacing angle and solve a system of six transcendental equations (50) relative to the unknowns $u_{1}, \vartheta_{1}, \varphi_{1}, u_{2}, \vartheta_{2}, \psi_{2}$. Taking into account that meshing equation $f\left(u_{1}, \vartheta_{1}, \varphi_{1}\right)=0$ is solved analytically (22) with respect to parameter $u_{1}$, five transcendental equations remain in the system (50).

The solution of the system (50) is performed numerically using the program developed in MathCad with $\psi_{1}=\psi_{1}^{*}=$ const . Finally, the values of parameters: $u_{1}^{*}, \vartheta_{1}^{*}, \varphi_{1}^{*}, u_{2}^{*}, \vartheta_{2}^{*}, \psi_{2}^{*}$ are determined, knowing which allows calculating the projection of the contact point on the wheel and pinion arc teeth flanks using formulas (42) and (44).

To calculate the loading of the contact in meshing arc teeth, it is necessary not only to have the coordinates of the contact point of their flanks, but also the values of the principal relative curvatures at this point. These curvatures characterize the size of the gap between the contacting flanks of the arc teeth up to the value of the second order of smallness in the differential neighborhood of the contact point.

The principal relative curvature in the longitudinal direction of the tooth $\left(k_{p 1}\right)$ based on the dependences (11) and (39) is calculated as follows:

$k_{p 1}=k_{21}-k_{11}$,

where: $k_{21}=-\cos \alpha_{0} /\left(u_{2}^{*} \cdot \sin \alpha_{0}-r_{g 2}\right)$.

To determine value $k_{11}$ by the expression (39), we use the formulas (37) and (38), in which we adopt $u=u_{1}^{*}, v=v_{1}^{*}$ and $\varphi_{2}=\varphi_{2}^{*}$.

The principal relative curvature in the profile direction of the tooth $\left(k_{p 2}\right)$ is the difference between $k_{22}=0$ and $k_{12}$ :

$k_{p 2}=k_{22}-k_{12}=-k_{12}$ 
where, by analogy with the expression (51), when calculating by the formulas (37), (38) and (39), we adopt values $k_{12}$ as follows: $u=u_{1}^{*}, v=v_{1}^{*}$, and $\varphi_{2}=\varphi_{2}^{*}$.

\section{Study of Geometric Characteristics of Semi-rolled Cylindrical Gear arc Teeth Meshing}

Based on the built mathematical models for calculating geometric characteristics of meshing semi-rolled cylindrical gear wheel and gear arc teeth, a program is developed in the MathCad software environment. We regard the results of the analysis of the active path of action position in meshing using this program on the example of the study of a semi-rolled cylindrical arc gear, which has the following parameters: $z_{1}^{*}=23 ; z_{2}^{*}=73$; normal modulus $m_{n}=10 \mathrm{~mm}$; tool displacement coefficients when cutting the teeth of the pinion $\chi_{1}=0,44$ and the wheel $\chi_{2}=0,042$; tooth width $b_{w}=120 \mathrm{~mm} ; \alpha_{0}=20^{\circ}$, radius of the pitch circle of the pinion $R_{w 1}=116,115 \mathrm{~mm}$ and the wheel $R_{w 2}=368,540 \mathrm{~mm}$, center distance $a_{w p}=a_{w s}=484,655 \mathrm{~mm}$. All the calculations are performed for two variants of contact localization in the longitudinal direction of the gear arc teeth. In the first variant (high localization), to cut the concave side of the arc pinion teeth and the convex side of the arc wheel teeth, circular cutting heads with calculated radii of $r_{g 1}=220 \mathrm{~mm}$ and $r_{g_{2}}=215 \mathrm{~mm}$ respectively are used. In the second variant (the contact is close to linear) they are $r_{g 1}=220 \mathrm{~mm}$ and $r_{g 2}=218 \mathrm{~mm}$ respectively.

The calculation of the points of the active path of action in meshing arc teeth and the principal relative curvatures in the longitudinal direction of the teeth at these points allows estimating the position and size of the contact pattern reflecting the contact point in the gear when generating the gear on the test machine [7]. Following the work [7], the contact pattern corresponds to the line of the level of the gap occurring in the vicinity of the contact point of the flanks and calculated by the formula:

$\Delta=0,006 \sqrt{m_{n}}$.

Value $\Delta$ is associated with $k_{p 1}$ by the relationship:

$\Delta=k_{p 1} \cdot z_{b}^{2} / 2$

where: $z_{b}$ is the half-width of the contact area. 
Combining (53) and (54), we obtain:

$z_{b}=\sqrt{2 \cdot \Delta / k_{p 1}}=\sqrt{\left(0.012 \sqrt{m_{n}}\right) / k_{p 1}}$.

Expression (55) allows calculating the half-length of the contact area in each phase of meshing the arc teeth and determining the size of the contact pattern relative to the contact point.

Figures 5-6 show the results of calculating the contact pattern for gear 1 and gear 2 in the presence of the teeth twist angle $\gamma=0.0015$.

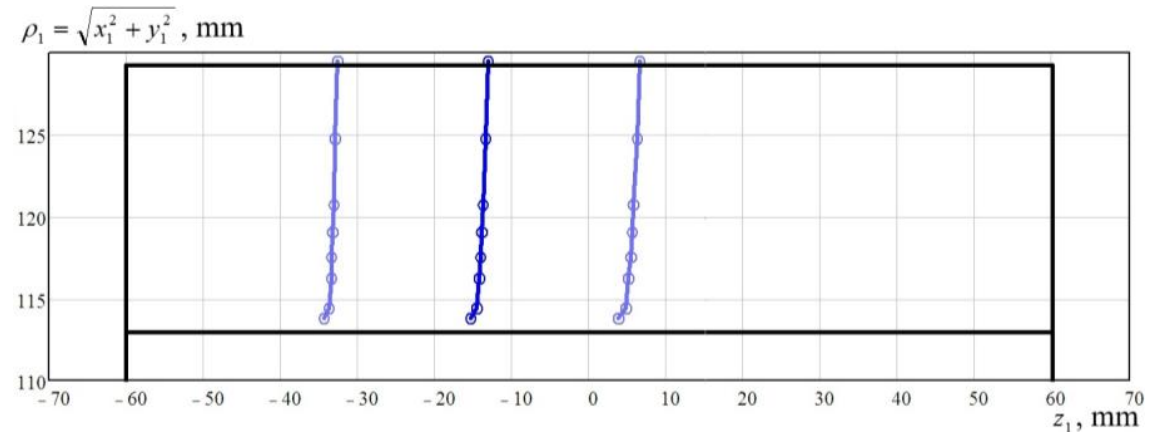

Figure 5

Active path of action and contact pattern in a cylindrical arc teeth gear (variant 1 ) at $\gamma=0.0015$

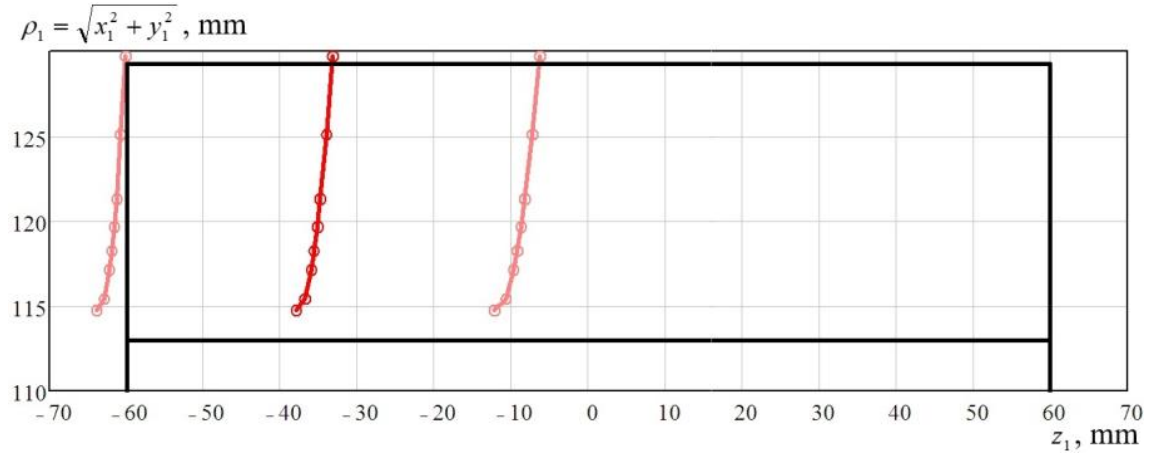

Figure 6

Active path of action and contact pattern in a cylindrical arc teeth gear (variant 2) at $\gamma=0.0015$

In the gear manufactured according to variant 2, the contact surface integrity of the pinion and wheel teeth is higher than in variant 1 . As a result, the width of the contact pattern has increased and is $\approx 53 \%$ in the absence of misalignment. At the same time, the sensitivity of the gear to the tooth flanks misalignment has increased. The left border of the contact pattern at a twist angle of $\gamma=0.0015$ already "goes" to the edge of the tooth (Figure 6), while the width of the contact pattern decreases by $\approx 20 \%$, which will cause an increase in contact stresses. 
At a twist angle of $\gamma=0.0021$, despite the presence of contact between the tooth surfaces, the width of the contact pattern is sharply reduced (by almost 50\%). The gear will operate essentially under edge contact conditions.

\section{Conclusions}

In this work, mathematical models of forming the gear wheel and pinion arc teeth flanks have been built. Dependences for calculating principal curvatures of the wheel and pinion arc teeth flanks have been obtained. An algorithm and a program for calculating the coordinates of the active path of action points in meshing arc teeth as well as principal relative curvatures at these points have been developed. The problem of determining the size of the contact pattern in the studied gear has been solved.

The calculated geometric characteristics of meshing gear arc teeth in the presence of a twist angle of the wheel and pinion teeth in meshing are the basis for calculating the loading capacity of the gear, which depends both on the position of the contact points of the teeth in meshing, principal relative curvatures at these points, and the maximum value of the twist angle of the teeth in meshing, when the performance and durability of the gear are to be provided.

\section{List of notations}

$S_{p}\left(x_{p}, y_{p}, z_{p}\right)$ - coordinate system rigidly connected to the cutting head;

$u, \vartheta$ - linear and angular parameters of the generating surface;

$\alpha_{0}$ - basic profile angle;

$r_{g 2}$ - calculated radius of the cutting head at wheel cutting;

$S_{2}\left(x_{2}, y_{2} z_{2}\right)$ - coordinate system rigidly connected to the wheel;

$\tilde{A}_{2, p}$ - matrix describing the transition from coordinate system $S_{p}\left(x_{p}, y_{p}, z_{p}\right)$ to system $S_{2}\left(x_{2}, y_{2} z_{2}\right)$;

$\bar{r}_{p}\left(x_{p}, y_{p}, z_{p}\right)$ - radius-vector of a generating surface;

$\bar{r}_{2}\left(x_{2}, y_{2}, z_{2}\right), \bar{m}_{2}\left(m_{2 x}, m_{2 y}, m_{2 z}\right)$ - radius vector and normal unitary vector of the arc wheel tooth flank;

$\tilde{r}_{2}, \tilde{r}_{p}-$ columns matrixes made up of coordinates of $\bar{r}_{2}\left(x_{2}, y_{2}, z_{2}\right)$ and $\bar{r}_{p}\left(x_{p}, y_{p}, z_{p}\right)$;

$R_{w 2}$ - radius of the wheel pitch circle;

$m_{2 x}, m_{2 y}, m_{2 z}$ - projections of the normal unitary vector of arc wheel tooth flank;

$d \bar{m}_{2}, d \bar{r}_{2}$-differentials of vectors $\bar{m}_{2}$ and $\bar{r}_{2}$;

$x_{2 u} ; z_{2 u} ; x_{2 \vartheta} ; z_{2 \vartheta} ; m_{2 x u} ; m_{2 x \vartheta} ; m_{2 z u} ; m_{2 z \vartheta}$ - partial derivatives of projections of coordinate of vectors $\bar{r}_{2}$ and $\bar{m}_{2}$ by parameters $u$ and $\vartheta$; 
$k_{21}, k_{22}$ - the principal curvatures of the arc wheel flank;

$z_{1}^{*}, z_{2}^{*}$ - the numbers of the pinion and wheel teeth;

$S_{1}\left(x_{1}, y_{1} z_{1}\right)$-coordinate system rigidly connected to the pinion;

$\varphi_{2}$ - the angle of the wheel rotation when forming the pinion tooth flank;

$\tilde{A}_{1,2}\left(\varphi_{2}\right)$ - matrix describing the transition from coordinate system $S_{2}\left(x_{2}, y_{2} z_{2}\right)$

to coordinate system $S_{1}\left(x_{1}, y_{1} z_{1}\right)$;

$a_{w s}$ - the center distance in the machine meshing of the pinion and the generating wheel;

$\bar{r}_{1}\left(x_{1}, y_{1}, z_{1}\right), \bar{m}_{1}\left(m_{1 x}, m_{1 y}, m_{1 z}\right)$ - radius vector and normal unitary vector of the arc pinion tooth flank;

$f\left(u, \vartheta, \varphi_{2}\right)=0$ the meshing equation;

$\bar{V}_{\varphi}$ - the vector analog of the relative speed;

$\tilde{C}_{\varphi}$ - the fourth-order matrix of the relative speed analog;

$d \bar{m}_{1}, d \bar{r}_{1}$ - differentials of vectors $\bar{m}_{1}$ and $\bar{r}_{1}$;

$f_{u}, f_{\vartheta}, f_{\varphi}$ - partial derivatives of the meshing $f\left(u, \vartheta, \varphi_{2}\right)=0$ by parameters

$u, \vartheta$ and $\varphi_{2}$;

$k_{11}, k_{12}$ - principal curvatures of the pinion tooth flank;

$\psi_{1}, \psi_{2}$ the angles of rotation of the pinion and wheel in working meshing;

$\gamma$ - twist angle of teeth in meshing;

$k_{p 1}, k_{p 2}$ - principal relative curvatures in arc teeth meshing;

$z_{b}$ - semi-length of contact pattern;

$r_{g 1}$ - calculated radius of the cutting head at pinion cutting.

\section{References}

[1] Syzrantsev V. N.: Cylindrical Arc Gears: History, Achievements, and Problems, Gears in Design, Production and Education. Mechanisms and Machine Science 101 (2021) 131-151, https://doi.org/10.1007/978-3-03073022-2 6

[2] Syzrantsev V., Syzrantseva K., Varshavsky M.: Contact Load and Endurance of Cylindrical Gearing with Arch-shaped Teeth, International Conference on Mechanical Transmissions (ICMT 2001), Chongqing, China (2001) 425-431 
[3] Arafa, H. A.: C-gears: Geometry and Machining, Proceedings of the Institution of Mechanical Engineers, Part C: Journal of Mechanical Engineering Science 219, Issue 7 (2005) 709-726

[4] Tsay G.-B., Fong Z. H.: Tooth Contact Analysis for Helical Gears with Pinion Circular Arc Teeth and Gear Involute Shaped Teeth, Journal of Mechanical Design, Transactions of the ASME 111, Issue 2 (1989) 278-284

[5] Tseng J.-T., Tsay C.-B.: Mathematical Model and Surface Deviation of Cylindrical Gears with Curvilinear Shaped Teeth Cut by Hob Cutter, Journal of Mechanical Design, Transactions of the ASME 127, Issue 5 (2005), 982-987

[6] Dai Y., Yukinori A., Jiang D.: Hobbing Mechanism of Cylindrical Gear with Arcuate Tooth Traces and Experimental Investigation, Zhongguo Jixie Gongcheng / China Mechanical Engineering, 17, Issue 7 (2006) 706-709

[7] Litvin F. L., Fuentes A.: Gear Geometry and Applied Theory, Cambridge: University Press, New York (2004) 800 p.

[8] Stadtfeld H. J.: Gleason Bevel Gear Technology. Manufacturing, Inspections and Optimization, The Gleason Works, Rochester, New York (1995) $202 \mathrm{p}$.

[9] Litvin F. L., Gutman, Y.: Methods of Synthesis and Analysis for Hypoid Gear Drives of Formate and Helixform. Parts 1, 2, and 3, ASME Journal of Mechanical Design 103, No. 1 (1981) 83-113

[10] Fan Q.: Computerized Modeling and Simulation of Spiral Bevel and Hypoid Gears Manufactured by Gleason Face Hobbing Process, Journal of Mechanical Design, Transactions of the ASME 128 (6) (2006) 1315-1327

[11] Syzrantseva K., Syzrantsev V., Babichev D.: Comparative Analysis of Stress-Strain Condition of Cylindrical Gears Arc Teeth and Spurs, Lecture Notes in Mechanical Engineering (2020) 101-108

[12] Syzrantseva K. V., Syzrantsev V. N., Kolbasin D. S.: Comparative Estimation of the Failure Probability of Cylindrical Arc and Helical Gears by Tooth Bending Endurance, AIP Conference Proceedings (2019) 2176:020010, doi.org/10.1063/1.5135122

[13] Syzrantsev V., Syzrantseva K., Kolbasin D.: Forming Surfaces of a Semirolled Cylindrical Gearing Wheel and a Gear Arc Teeth, Lecture Notes in Mechanical Engineering (2021) 134-141, https://doi.org/10.1007/978-3030-54814-8_16

[14] Syzrantsev Vladimir, Syzrantseva Ksenia: Study of Geometric Characteristics of the Arc Teeth Semi-rolled Cylindrical Gear Meshing, FME Transactions 49 (2021) 367-373 doi:10.5937/fme2102367S

[15] Bálint Laczik, Peter Zentay, Richárd Horváth: A New Approach for Designing Gear Profiles using Closed Complex Equations, Acta Polytechnica Hungarica 11 (2014) 159-172 